\title{
Does the three site Higgsless model survive the electroweak precision tests at loop?
}

\author{
Tomohiro Abe, ${ }^{1}$ Shinya Matsuzaki ${ }^{2}$ and Masaharu Tanabashi ${ }^{1}$ \\ 1 Department of Physics, Nagoya University, Nagoya 464-8602, Japan \\ ${ }^{2}$ Department of Physics and Astronomy, University of North Carolina, Chapel Hill, NC 27599, USA
}

(Dated: October 28, 2018)

\begin{abstract}
We complete the list of one loop renormalization group equations and matching conditions relevant for the computation of the electroweak precision parameters $S$ and $T$ in the three site Higgsless model. We obtain one-loop formulas for $S$ and $T$ expressed in terms of physical observables such as the KK gauge boson mass $M_{W^{\prime}}$, the KK fermion mass $M$, and the KK gauge boson $\left(W^{\prime}\right)$ couplings with light quarks and leptons $g_{W^{\prime} f f}$. It is shown that these physical observables, $M_{W^{\prime}}, M$ and $g_{W^{\prime} f f}$ are severely constrained by the electroweak precision data. Unlike the tree level analysis on the ideally delocalized fermion, we find that perfect fermiophobity of $W^{\prime}$ is ruled out by the precision data. We also study the cutoff dependence of our analysis. Although the model is nonrenormalizable, the dependence on the cutoff parameter $\Lambda$ is shown to be non-significant.

PACS numbers: $12.60 . \mathrm{Cn}, 12.15 \mathrm{Lk}$
\end{abstract}

\section{INTRODUCTION}

Does a scalar Higgs boson necessarily exist as required in the standard model? Higgsless models 1] provide a negative answer to this question, achieving the electroweak symmetry breaking (EWSB) without invoking an elementary Higgs particle[2]. The unitarity of longitudinally polarized $W$ and $Z$ boson scattering is effectively maintained beyond the $\mathrm{TeV}$ energy scale by the exchange of an infinite tower of massive spin-1 particles [3, 4, 5, 6], rather than a spinless Higgs boson. These massive spin-1 particles may be provided as Kaluza-Klein $(\mathrm{KK})$ modes $\left(W^{\prime}, W^{\prime \prime}, W^{\prime \prime \prime} \cdots, Z^{\prime}\right.$, $Z^{\prime \prime}, Z^{\prime \prime \prime} \ldots$ ) of five dimensional gauge fields compactified on a TeV scale interval with appropriate boundary conditions 7, 8, 9, 10]. Deconstruction [11, 12] of extra dimensions enables us to construct four dimensional gauge invariant phenomenological models which approximate the five dimensional Higgsless theories. The deconstructed Higgsless models [13, 14, 15, 16, 17, 18, 19] (or linear Moose 20] models) can be used as tools to compute the low energy properties of Higgsless theories below the cutoff scale.

Higgsless models are often regarded as "dual" 21, 22, 23, 24] to models of dynamical EWSB 25, 26] such as "walking technicolor" 27, 28, 29, 30, 31, 32]. The deconstructed version of the Higgsless model possesses extended electroweak gauge symmetries, which can be thought as analogues of hidden local symmetries (HLS) 33, 34, 35, 36, 37, 38] and the vector limit symmetry [39] in the dynamical chiral symmetry breaking of QCD. The gauge sector of the Higgsless model therefore has some similarities to the BESS models [40, 41].

Any phenomenologically viable EWSB model should satisfy constraints from the precision electroweak parameters 42, 43, 44]. An analysis in a very general class of linear Moose model shows that a Higgsless model with brane localized fermion cannot satisfy simultaneously both unitarity bounds and precision electroweak constraints at tree level. 19] Delocalizing fermions within the extra dimension may reduce significantly the sizes of electroweak corrections. 45, 46, 47, 48. In the deconstruction language the delocalizing fermions may be achieved by allowing fermions to derive electroweak properties from more than one site over the deconstruction lattice. [49, 50, 51] It has been shown that, for an arbitrary Higgsless model with "ideally" delocalized fermions [51], three $(\hat{S}, \hat{T}, W)$ of the leading zeromomentum precision electroweak parameters defined by Barbieri et al. [52, 53] vanish at tree level. Moreover, the ideal delocalization implies fermiophobic $W^{\prime}$. Presently existing direct $W^{\prime}$ searches cannot be applied for such a fermiophobic $W^{\prime}$ boson. The strongest constraints on the $W^{\prime}$ and $Z^{\prime}$ masses in the Higgsless models then come from the limits on deviations in multi-gauge-boson vertices. [54, 55]

Recently, a three site Higgsless model[56] has been proposed. This theory incorporates only three sites on the deconstruction lattice, and may be regarded as the simplest deconstructed Higgsless model. The three site model possesses the same gauge group structure as that of the BESS model [40, 41]. The new interest is aroused in its fermion sector such as the fermion delocalization. The fermionic loop correction to the $\rho$ parameter ( $T$ parameter) has been calculated in the Higgsless model. 56, 57| With the aid of electroweak chiral Lagrangian [58, 59, 60, 61, 62, 63], and using the technique described in 37, 38, the bosonic chiral logarithmic loop corrections 64, 65] to the electroweak precision parameters have been evaluated in [66, 67, 68, 69].

In this paper, we further investigate the structure of one loop radiative corrections in the three site Higgsless model, and present the complete list of one loop renormalization group equations and matching conditions relevant for the computation of $S$ and $T$ including all of fermionic- and bosonic-loop corrections. We obtain oneloop formulas $S$ and $T$ expressed in terms of physical observables such as the KK gauge boson mass $M_{W^{\prime}}$, the KK fermion mass $M$, and the $W^{\prime}$ couplings with light quarks and leptons $g_{W^{\prime} f f}$. It is shown that the $W^{\prime} f f$ 
couplings and the KK fermion mass are severely constrained by the electroweak precision parameters. Unlike the tree level analysis on the ideally delocalized fermion, we find that the $W^{\prime}$ boson needs to have non-vanishing coupling with light fermions in order to satisfy the precision electroweak constraints. The dependence on the ultraviolet cutoff parameter is also studied. In spite of the non-renormalizability of the model, we find the cutoff dependence is not significant in the numerical analysis.

The next section of the paper introduces the gauge sector Lagrangian, the symmetry breaking sector Lagrangian, and the fermion sector Lagrangian. We present the fermion sector Lagrangian above the KK fermion mass scale $M$ and obtain the effective fermion sector Lagrangian after integrating out the KK fermion fields below $M$. A new operator $x_{1}^{\prime}$, which affects the on-shell $W^{\prime} f f$ coupling strength, needs to be introduced to absorb the one loop divergence. In Section III, we summarize the renormalization group equations relevant for the computation of $S$ and $T$ parameters. Section IV is for the matching conditions to the electroweak chiral Lagrangian. Section $\mathrm{V}$ then gives our final results on the $S$ and $T$ parameters expressed in terms of physical observables. We then compare our formulas with the precision electroweak data in Section VI, and find that the $W^{\prime} f f$ couplings and the KK fermion mass are severely bounded. Especially, it is shown that the precision electroweak data reject the perfect fermiophobity of the $W^{\prime}$ boson. Section VII summarizes the results of our analysis. The renormalization group equation of the operator $x_{1}^{\prime}$ is derived in the Appendix A.

\section{THREE SITE HIGGSLESS MODEL}

In this section we make a brief review on the three site Higgsless model[56]. Higher order counter terms, as well as the lowest order Lagrangian terms, are introduced so as to subtract one-loop divergences.

\section{A. Gauge symmetry and its breaking}

We first consider the gauge sector Lagrangian in the three site Higgsless model. The Moose diagram of this model is illustrated in Figure 1. The model incorporates $S U(2) \times S U(2) \times U(1)$ gauge fields $V_{i \mu}(i=0,1,2)$ and two $S U(2)$ non-linear sigma model fields $U_{i}(i=1,2)$. The VEVs $\left\langle U_{1}\right\rangle$ and $\left\langle U_{2}\right\rangle$ break the gauge symmetry down to $U(1)_{e m}$. The model therefore possesses $W^{\prime}$ and $Z^{\prime}$ bosons, identified as the KK gauge bosons, in addition to the usual $W, Z$ and photon. The corresponding lowest order Lagrangian $\mathcal{L}_{2}$ is given by

$$
\mathcal{L}_{2}=\sum_{i=1}^{2} \frac{f_{i}^{2}}{4} \operatorname{tr}\left[\left(D_{\mu} U_{i}\right)^{\dagger}\left(D^{\mu} U_{i}\right)\right]-\sum_{i=0}^{2} \frac{1}{2 g_{i}^{2}} \operatorname{tr}\left[V_{i \mu \nu} V_{i}^{\mu \nu}\right],
$$

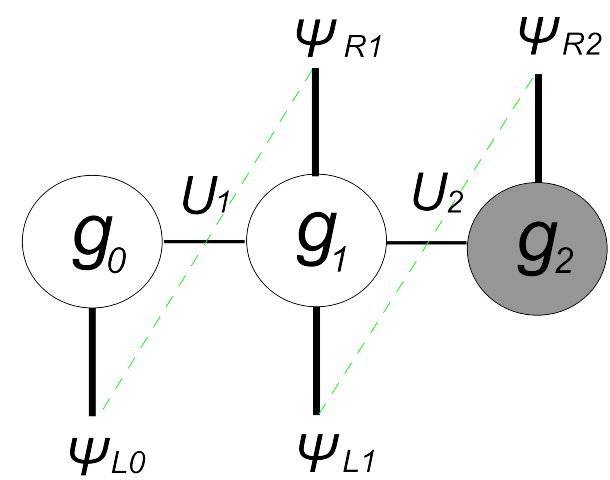

FIG. 1: The three site Higgsless Moose diagram. Sites 0 and 1 represent $S U(2)$ gauge fields with coupling strengths $g_{0}$ and $g_{1}$. Site 2 corresponds to a $U(1)$ gauge field with coupling strength $g_{2}$. The non-linear sigma model field $U_{1}$ $\left(U_{2}\right)$ is denoted by link between sites 0 and 1 (sites 1 and 2). The left-handed fermions $\psi_{L 0}$ and $\psi_{L 1}$ feel the gauge fields at sites 0 and 1 , while the right-handed fermions $\psi_{R 1}$ and $\psi_{R 2}$ at sites 1 and 2. The dashed green lines represent corresponding Yukawa couplings.

where $D_{\mu} U_{i}$ and $V_{i \mu \nu}$ are defined as

$$
\begin{gathered}
D_{\mu} U_{i} \equiv \partial_{\mu} U_{i}+i V_{(i-1) \mu} U_{i}-i U_{i} V_{i \mu}, \\
V_{i \mu \nu} \equiv \partial_{\mu} V_{i \nu}-\partial_{\nu} V_{i \mu}+i\left[V_{i \mu}, V_{i \nu}\right] .
\end{gathered}
$$

The first two gauge fields $(i=0,1)$ correspond to $S U(2)$ gauge groups,

$$
V_{i \mu}=\sum_{a=1,2,3} \frac{\tau^{a}}{2} V_{i \mu}^{a}, \quad \text { for } i=0,1
$$

while the last gauge field $(i=2)$ corresponds to $U(1)$ group embedded as the $T_{3}$ generator of $S U(2)$,

$$
V_{2 \mu}=\frac{\tau^{3}}{2} V_{2 \mu}^{3}, \quad \text { for } i=2
$$

The violation of the global $S U(2)$ invariance in Eq.(II.5) causes an $S U(2)$ violating one-loop divergence. We subtract this divergence by introducing a counter term [67],

$$
\mathcal{L}_{2}^{\prime}=\beta_{(2)} \frac{f_{2}^{2}}{4} \operatorname{tr}\left[U_{2}^{\dagger}\left(D_{\mu} U_{2}\right) \tau^{3}\right] \operatorname{tr}\left[U_{2}^{\dagger}\left(D^{\mu} U_{2}\right) \tau^{3}\right] .
$$

The counter term Eq.(II.6) is of $\mathcal{O}\left(p^{2}\right)$ in the usual chiral perturbation order counting rule 64, 65. This situation is similar to the custodial $S U(2)$ symmetry violation in the electroweak chiral Lagrangian. [58, 59, 60, 61, 62, 63].

Other one-loop divergences arising from the lowest order Lagrangian Eq.(II.1) are invariant under the global $S U(2)$ symmetry. In order to subtract these $S U(2)$ invariant one-loop divergences, we introduce appropriate 
counter terms $[67]^{1}$

$$
\mathcal{L}_{4}=\sum_{i=1}^{2} \mathcal{L}_{4}^{(i)}
$$

with

$$
\begin{aligned}
\mathcal{L}_{4}^{(i)}= & \alpha_{(i) 1} \operatorname{tr}\left[V_{(i-1) \mu \nu} U_{i} V_{i}^{\mu \nu} U_{i}^{\dagger}\right] \\
& -2 i \alpha_{(i) 2} \operatorname{tr}\left[\left(D_{\mu} U_{i}\right)^{\dagger}\left(D_{\nu} U_{i}\right) V_{i}^{\mu \nu}\right] \\
& -2 i \alpha_{(i) 3} \operatorname{tr}\left[V_{(i-1)}^{\mu \nu}\left(D_{\mu} U_{i}\right)\left(D_{\nu} U_{i}\right)^{\dagger}\right] \\
& +\alpha_{(i) 4} \operatorname{tr}\left[\left(D_{\mu} U_{i}\right)\left(D_{\nu} U_{i}\right)^{\dagger}\right] \operatorname{tr}\left[\left(D^{\mu} U_{i}\right)\left(D^{\nu} U_{i}\right)^{\dagger}\right] \\
& +\alpha_{(i) 5} \operatorname{tr}\left[\left(D_{\mu} U_{i}\right)\left(D^{\mu} U_{i}\right)^{\dagger}\right] \operatorname{tr}\left[\left(D_{\nu} U_{i}\right)\left(D^{\nu} U_{i}\right)^{\dagger}\right] .
\end{aligned}
$$

These terms are of $\mathcal{O}\left(p^{4}\right)$ in the conventional chiral perturbation order counting rule. Again, the situation is similar to the electroweak chiral Lagrangian.

Reflecting its coarse deconstruction, the delay of unitarity violation is shown to be modest in the lowest order three site model Eq.(II.1): the unitarity in the elastic $W_{L} W_{L}$ scattering amplitude is violated at $2 \mathrm{TeV}$ for $f_{1}=f_{2}$ with this setup. 70] It is expected, however, if the higher order operators Eq.(II.8) are included in the analysis, unitarity may hold up to a higher energy scale.

\section{B. Fermion sector above $M$}

We next turn to the fermion sector. Quantum numbers of quark/lepton fields are summarized in Table [. The superscript $n=1,2,3$ specifies the generation of quarks and leptons. The chiralities are denoted by subscript $L$ and $R$. Fields $q_{L}^{n, 0}$ and $\ell_{L}^{n, 0}$, denoted as $\psi_{L 0}$ in Figure 1 . live on the site 0 in the Moose diagram, while $q_{L, R}^{n, 1}$ and $\ell_{L, R}^{n, 1}$, denoted as $\psi_{L 1}$ and $\psi_{R 1}$ in Figure 1, are on the site 1 . The site 2 fermion field $\psi_{R 2}$ denotes $u_{R}^{n, 2}, d_{R}^{n, 2}$ and $e_{R}^{n, 2}$. This model incorporates heavier KK-fermions as well as the ordinary light quarks and leptons. The kinetic term Lagrangian for these fermion fields is given by

$$
\begin{aligned}
\mathcal{L}_{\text {kinetic }}= & \sum_{i=0,1} \sum_{n} \bar{q}_{L}^{n, i} i \not D q_{L}^{n, i}+\sum_{n} \bar{q}_{R}^{n, 1} i \not D q_{R}^{n, 1} \\
& +\sum_{n} \bar{u}_{R}^{n, 2} i \not D u_{R}^{n, 2}+\sum_{n} \bar{d}_{R}^{n, 2} i \not D d_{R}^{n, 2} \\
& +\sum_{i=0,1} \sum_{n} \bar{\ell}_{L}^{n, i} i \not D \ell_{L}^{n, i}+\sum_{n} \bar{\ell}_{R}^{n, 1} i \not D \ell_{R}^{n, 1} \\
& +\sum_{n} \bar{e}_{R}^{n, 2} i \not D e_{R}^{n, 2} .
\end{aligned}
$$

\footnotetext{
1 We assume here an approximate global $S U(2)$ symmetry at the cutoff scale. See Ref. [67] for a complete list of $\mathcal{O}\left(p^{4}\right)$ counter terms including $S U(2)$ violating operators.
}

$S U(3)_{c} S U(2)_{0} S U(2)_{1} \quad U(1)_{2}$

\begin{tabular}{c|cccc}
\hline$q_{L}^{n, 0}$ & $\underline{3}$ & $\underline{2}$ & $\underline{1}$ & $1 / 6$ \\
$\ell_{L}^{n, 0}$ & $\underline{1}$ & $\underline{2}$ & $\underline{1}$ & $-1 / 2$ \\
$q_{L}^{n, 1}$ & $\underline{3}$ & $\underline{1}$ & $\underline{2}$ & $1 / 6$ \\
$\ell_{L}^{n, 1}$ & $\underline{1}$ & $\underline{1}$ & $\underline{2}$ & $-1 / 2$ \\
$q_{R}^{n, 1}$ & $\underline{3}$ & $\underline{1}$ & $\underline{2}$ & $1 / 6$ \\
$\ell_{R}^{n, 1}$ & $\underline{1}$ & $\underline{1}$ & $\underline{2}$ & $-1 / 2$ \\
$u_{R}^{n, 2}$ & $\underline{3}$ & $\underline{1}$ & $\underline{1}$ & $2 / 3$ \\
$d_{R}^{n, 2}$ & $\underline{3}$ & $\underline{1}$ & $\underline{1}$ & $-1 / 3$ \\
$e_{R}^{n, 2}$ & $\underline{1}$ & $\underline{1}$ & $\underline{1}$ & -1
\end{tabular}

TABLE I: Quantum numbers of quark/lepton fields. The superscript $n$ specifies the generation of quarks and leptons. The chiralities are denoted by subscripts $L$ and $R$.

Mass terms of these fermion fields are assumed to be

$$
\begin{aligned}
\mathcal{L}_{\text {mass }}= & -m_{1} \sum_{n} \bar{q}_{L}^{n, 0} U_{1} q_{R}^{n, 1}-m_{1} \sum_{n} \bar{\ell}_{L}^{n, 0} U_{1} \ell_{R}^{n, 1} \\
& -M \sum_{n} \bar{q}_{L}^{n, 1} q_{R}^{n, 1}-M \sum_{n} \bar{\ell}_{L}^{n, 1} \ell_{R}^{n, 1}+\text { h.c. }
\end{aligned}
$$

and

$$
\mathcal{L}_{\text {mass }}^{\prime}=-m_{t}^{\prime} \bar{q}_{L}^{3,1} U_{2} P_{t}\left(\begin{array}{c}
t_{R}^{2} \\
b_{R}^{2}
\end{array}\right)+\text { h.c., } \quad P_{t} \equiv\left(\begin{array}{cc}
1 & 0 \\
0 & 0
\end{array}\right),
$$

with $t_{R}^{2}$ and $b_{R}^{2}$ being defined by

$$
t_{R}^{2} \equiv u_{R}^{3,2}, \quad b_{R}^{2} \equiv d_{R}^{3,2} .
$$

Here, for simplicity, we incorporate Yukawa coupling of $U_{2}$ only for the top quark. The other light quarks and leptons thus remain massless in this setup. It is straightforward to obtain realistic masses of quarks and leptons (and CKM mixings) by allowing more general Yukawa couplings with $U_{2}$, however. We also assumed $m_{1}$ and $M$ are $n$-independent (generation independent). This ensures the suppression of FCNC through the GIM mechanism even when we introduce more general $U_{2}$ Yukawa couplings. We assumed $m_{1}$ and $M$ are quark-lepton universal. As we will discuss later, the ratio $m_{1} / M$ affects the $W$ couplings with ordinary light quarks and leptons. The universal $m_{1} / M$ thus guarantees the quark-lepton universality of the $W$ couplings.

In order to renormalize the one loop divergences arising from the Yukawa couplings in Eq.(II.10), we need to introduce

$$
\begin{aligned}
\mathcal{L}_{f}= & -x_{1} \sum_{n} \bar{q}_{L}^{n, 0} i \gamma^{\mu}\left(D_{\mu} U_{1}\right) U_{1}^{\dagger} q_{L}^{n, 0} \\
& -x_{1} \sum_{n} \bar{\ell}_{L}^{n, 0} i \gamma^{\mu}\left(D_{\mu} U_{1}\right) U_{1}^{\dagger} \ell_{L}^{n, 0} \\
& +\tilde{x}_{1} \sum_{n} \bar{q}_{R}^{n, 1} i \gamma^{\mu} U_{1}^{\dagger}\left(D_{\mu} U_{1}\right) q_{R}^{n, 1} \\
& +\tilde{x}_{1} \sum_{n} \bar{\ell}_{R}^{n, 1} i \gamma^{\mu} U_{1}^{\dagger}\left(D_{\mu} U_{1}\right) \ell_{R}^{n, 1} .
\end{aligned}
$$


We also need to introduce

$$
\begin{aligned}
\mathcal{L}_{f}^{\prime}= & -x_{2} \bar{q}_{L}^{3,1} i \gamma^{\mu}\left(D_{\mu} U_{2}\right) U_{2}^{\dagger} q_{L}^{3,1} \\
& -x_{23} \bar{q}_{L}^{3,1} i \gamma^{\mu} q_{L}^{3,1} \operatorname{tr}\left[\tau^{3} U_{2}^{\dagger} D_{\mu} U_{2}\right] \\
& +\tilde{x}_{2}\left(\bar{t}_{R}^{2}, \bar{b}_{R}^{2}\right) i \gamma^{\mu} P_{t} U_{2}^{\dagger}\left(D_{\mu} U_{2}\right) P_{t}\left(\begin{array}{c}
t_{R}^{2} \\
b_{R}^{2}
\end{array}\right) \\
& -\Delta M_{t} \bar{q}_{L}^{3,1} U_{2} P_{t} U_{2}^{\dagger} q_{R}^{3,1} \\
& -\Delta M_{t} \bar{q}_{R}^{3,1} U_{2} P_{t} U_{2}^{\dagger} q_{L}^{3,1},
\end{aligned}
$$

for the renormalization of the one loop divergences arising from the Yukawa coupling in Eq.(II.11).

\section{Fermion sector below $M$}

We assume

$$
M \gg m_{1}, m_{t}^{\prime} \text {. }
$$

The KK fermion mass is well approximated by the Dirac mass $M$ in this limit. We see that the KK fermions are largely made from $q_{L, R}^{n, 1}$ and $\ell_{L, R}^{n, 1}$. It is easy to integrate out these KK fermion fields $q_{L, R}^{n, 1}$ and $\ell_{L, R}^{n, 1}$. The low energy effective theory below $M$ is described by

$$
\begin{aligned}
\mathcal{L}_{\text {mass }}= & -m_{t} \bar{q}_{L}^{3,0} U_{1} U_{2} P_{t}\left(\begin{array}{c}
t_{R}^{2} \\
b_{R}^{2}
\end{array}\right), \\
\mathcal{L}_{f}= & -\sum_{n} x_{1} \bar{q}_{L}^{n, 0} i\left(D_{\mu} U_{1}\right) U_{1}^{\dagger} \gamma^{\mu} q_{L}^{n, 0} \\
& -\sum_{n} x_{1} \bar{\ell}_{L}^{n, 0} i\left(D_{\mu} U_{1}\right) U_{1}^{\dagger} \gamma^{\mu} \ell_{L}^{n, 0},
\end{aligned}
$$

and

$$
\mathcal{L}_{f}^{\prime}=\tilde{x}_{2}\left(\bar{t}_{R}^{2}, \bar{b}_{R}^{2}\right) i \gamma^{\mu} P_{t} U_{2}^{\dagger}\left(D_{\mu} U_{2}\right) P_{t}\left(\begin{array}{c}
t_{R}^{2} \\
b_{R}^{2}
\end{array}\right)
$$

in addition to the light fermion kinetic terms and the gauge sector Lagrangians Eqs.(II.1), (II.6) and (II.8).

In order to renormalize the one-loop divergences arising from the fermion Lagrangian Eq.(II.17), we introduce counter terms

$$
\frac{f_{12}^{2}}{4} \operatorname{tr}\left[\left(D_{\mu}\left(U_{1} U_{2}\right)\right)\left(D^{\mu}\left(U_{1} U_{2}\right)\right)^{\dagger}\right]
$$

and

$$
\begin{aligned}
& -\sum_{n} x_{1}^{\prime} \bar{q}_{L}^{n, 0}\left[D^{\nu}\left(U_{1} V_{1 \mu \nu} U_{1}^{\dagger}\right)\right] \gamma^{\mu} q_{L}^{n, 0} \\
& -\sum_{n} x_{1}^{\prime} \bar{\ell}_{L}^{n, 0}\left[D^{\nu}\left(U_{1} V_{1 \mu \nu} U_{1}^{\dagger}\right)\right] \gamma^{\mu} \ell_{L}^{n, 0} .
\end{aligned}
$$

At the scale $\mu=M$, these parameters are determined as

$$
f_{12}^{2}(\mu=M) \simeq 0
$$

and

$$
x_{1}^{\prime}(\mu=M) \simeq 0 .
$$

Note that the operator $x_{1}^{\prime}$ leads to non-trivial corrections to the $W^{\prime}$ couplings with light fermions at the mass-shell of the $W^{\prime}$ boson.

\section{RENORMALIZATION GROUP EQUATIONS}

We are now ready to discuss one-loop ultraviolet divergences arising from the lowest order Lagrangian. Similarly to the usual chiral perturbation theory [64, 65], these divergences can be subtracted through the renormalization of higher order counter terms. The renormalized parameters then depend on a choice of the renormalization scale $\mu$. The renormalization group equations arising from the bosonic chiral loop corrections have already been reported in Ref.[67]. In this section, we report our newly derived results on the fermionic loop diagrams in the three site Higgsless model, as well as the bosonic loop diagrams.

\section{A. Above KK fermion mass $M$}

Performing the standard $\overline{\mathrm{MS}}$ renormalization, we find

$$
\begin{aligned}
\mu \frac{d}{d \mu} f_{1}^{2} & =\frac{3}{(4 \pi)^{2}}\left(g_{0}^{2}+g_{1}^{2}\right) f_{1}^{2}-\frac{1}{2 \pi^{2}} N_{g}\left(N_{c}+1\right) m_{1}^{2}, \\
\mu \frac{d}{d \mu} f_{2}^{2} & =\frac{3}{(4 \pi)^{2}}\left(g_{1}^{2}+\frac{1}{2} g_{2}^{2}\right) f_{2}^{2}-\frac{1}{4 \pi^{2}} N_{c} m_{t}^{\prime 2}, \text { (III.2) } \\
\mu \frac{d}{d \mu}\left(\beta_{(2)} f_{2}^{2}\right) & =\frac{3}{4(4 \pi)^{2}} g_{2}^{2} f_{2}^{2},
\end{aligned}
$$

for $\mathcal{O}\left(p^{2}\right)$ parameters, and

$$
\begin{aligned}
& \mu \frac{d}{d \mu}\left(\frac{1}{g_{0}^{2}}\right)=\frac{1}{(4 \pi)^{2}}\left[\frac{44}{3}-\frac{1}{6}-\frac{2}{3} N_{g}\left(N_{c}+1\right)\right], \\
& \mu \frac{d}{d \mu}\left(\frac{1}{g_{1}^{2}}\right)=\frac{1}{(4 \pi)^{2}}\left[\frac{44}{3}-\frac{2}{6}-\frac{4}{3} N_{g}\left(N_{c}+1\right)\right], \\
& \mu \frac{d}{d \mu}\left(\frac{1}{g_{2}^{2}}\right)=\frac{1}{(4 \pi)^{2}}\left[-\frac{1}{6}-\frac{4}{3} N_{g}\left(\frac{13}{18} N_{c}+\frac{5}{2}\right)\right], \\
& \mu \frac{d}{d \mu}\left(\frac{1}{g_{s}^{2}}\right)=\frac{1}{(4 \pi)^{2}}\left[22-\frac{16}{3} N_{g}\right],
\end{aligned}
$$

for gauge coupling strengths. Here $N_{g}$ and $N_{c}$ denote the numbers of generations and colors, respectively,

$$
N_{g}=3, \quad N_{c}=3,
$$

and the $S U(3)_{c}$ gauge coupling is denoted by $g_{s}$. 
We also obtain 67 .

$$
\begin{aligned}
\mu \frac{d}{d \mu} \alpha_{(i) 1} & =\frac{1}{6(4 \pi)^{2}} \\
\mu \frac{d}{d \mu} \alpha_{(i) 2} & =\frac{1}{12(4 \pi)^{2}} \\
\mu \frac{d}{d \mu} \alpha_{(i) 3} & =\frac{1}{12(4 \pi)^{2}} \\
\mu \frac{d}{d \mu} \alpha_{(i) 4} & =-\frac{1}{6(4 \pi)^{2}} \\
\mu \frac{d}{d \mu} \alpha_{(i) 5} & =-\frac{1}{12(4 \pi)^{2}}
\end{aligned}
$$

for $\mathcal{O}\left(p^{4}\right)$ terms. We assumed $\beta_{(2)} \ll 1$ in these expression. Effects of $\mathcal{O}\left(\beta_{(2)}\right)$ are suppressed in the RHSs of these renormalization group equations. The bosonic loop terms in these expressions have already been reported in Ref. [67]. The fermionic loop effects, proportional to $N_{g}$ and/or $N_{c}$, are newly derived in the present study.

The Dirac mass $M$, the Yukawa couplings $m_{1}$ and $m_{t}^{\prime}$ also depend on the renormalization scale $\mu$. For the third generation quarks, we find

$$
\begin{array}{ll}
\mu \frac{d}{d \mu} m_{1}=\frac{m_{1}}{(4 \pi)^{2}}\left[-8 g_{s}^{2}-\frac{1}{6} g_{2}^{2}-3 \frac{m_{1}^{2}}{f_{1}^{2}}\right], & \text { (III.14) } \\
\mu \frac{d}{d \mu} M=\frac{M}{(4 \pi)^{2}}\left[-8 g_{s}^{2}-\frac{9}{2} g_{1}^{2}-\frac{1}{6} g_{2}^{2}+\frac{3}{2} \frac{m_{1}^{2}}{f_{1}^{2}}+\frac{m_{t}^{\prime 2}}{f_{2}^{2}}\right], \\
\mu \frac{d}{d \mu} m_{t}^{\prime}=\frac{m_{t}^{\prime}}{(4 \pi)^{2}}\left[-8 g_{s}^{2}-\frac{2}{3} g_{2}^{2}\right] .
\end{array}
$$

Combining Eq.(III.14) and Eq.(III.15), we obtain a renormalization group equation for the ratio $m_{1} / M$.

$$
\mu \frac{d}{d \mu}\left(\frac{m_{1}}{M}\right)=\frac{1}{(4 \pi)^{2}} \frac{m_{1}}{M}\left(\frac{9}{2} g_{1}^{2}-\frac{9}{2} \frac{m_{1}^{2}}{f_{1}^{2}}-\frac{m_{t}^{\prime 2}}{f_{2}^{2}}\right) .
$$

In a similar manner, we find

$$
\begin{aligned}
\mu \frac{d}{d \mu} m_{1} & =\frac{m_{1}}{(4 \pi)^{2}}\left[-8 g_{s}^{2}-\frac{1}{6} g_{2}^{2}-3 \frac{m_{1}^{2}}{f_{1}^{2}}\right], \\
\mu \frac{d}{d \mu} M & =\frac{M}{(4 \pi)^{2}}\left[-8 g_{s}^{2}-\frac{9}{2} g_{1}^{2}-\frac{1}{6} g_{2}^{2}+\frac{3}{2} \frac{m_{1}^{2}}{f_{1}^{2}}\right],
\end{aligned}
$$

for the first and second generation quark fields, and

$$
\begin{aligned}
\mu \frac{d}{d \mu} m_{1} & =\frac{m_{1}}{(4 \pi)^{2}}\left[-\frac{3}{2} g_{2}^{2}-3 \frac{m_{1}^{2}}{f_{1}^{2}}\right] \\
\mu \frac{d}{d \mu} M & =\frac{M}{(4 \pi)^{2}}\left[-\frac{9}{2} g_{1}^{2}-\frac{3}{2} g_{2}^{2}+\frac{3}{2} \frac{m_{1}^{2}}{f_{1}^{2}}\right]
\end{aligned}
$$

for lepton fields. Note that the renormalization group equations of the lepton mass terms Eqs.(III.20), (III.21) differ from those of the quark mass terms Eqs.(III.18), (III.19). The renormalization group equation for the ratio $m_{1} / M$

$$
\mu \frac{d}{d \mu}\left(\frac{m_{1}}{M}\right)=\frac{1}{(4 \pi)^{2}} \frac{m_{1}}{M}\left(\frac{9}{2} g_{1}^{2}-\frac{9}{2} \frac{m_{1}^{2}}{f_{1}^{2}}\right)
$$

is, on the other hand, universal to all fermions other than the third generation quarks. An implication of this fact will be discussed in the next subsection.

We next summarize the renormalization group equations for the one-loop generated operators listed in Eq.(II.13). We find

$$
\begin{aligned}
& \mu \frac{d}{d \mu} x_{1}=-\frac{1}{(4 \pi)^{2}} \frac{m_{1}^{2}}{f_{1}^{2}}, \\
& \mu \frac{d}{d \mu} \tilde{x}_{1}=-\frac{1}{(4 \pi)^{2}} \frac{m_{1}^{2}}{f_{1}^{2}} .
\end{aligned}
$$

In a similar manner, the top quark Yukawa coupling $m_{t}^{\prime}$ generates

$$
\begin{aligned}
\mu \frac{d}{d \mu} x_{2} & =-\frac{1}{2(4 \pi)^{2}} \frac{m_{t}^{\prime 2}}{f_{2}^{2}}, \\
\mu \frac{d}{d \mu} x_{23} & =-\frac{1}{4(4 \pi)^{2}} \frac{m_{t}^{\prime 2}}{f_{2}^{2}} \\
\mu \frac{d}{d \mu} \tilde{x}_{2} & =-\frac{1}{(4 \pi)^{2}} \frac{m_{t}^{\prime 2}}{f_{2}^{2}}, \\
\mu \frac{d}{d \mu}\left(\Delta M_{t}\right) & =-\frac{M}{2(4 \pi)^{2}} \frac{m_{t}^{\prime 2}}{f_{2}^{2}},
\end{aligned}
$$

at the one loop level.

\section{B. Matching at the KK fermion mass scale $M$}

The effective theory parameters in Eqs.(II.16)-(II.18) can be determined through the matching conditions at the KK fermion mass scale $M$,

$$
\begin{gathered}
x_{1}(\mu=M-0) \simeq x_{1}(\mu=M+0)+\left.\frac{m_{1}^{2}}{M^{2}}\right|_{\mu=M}, \\
\left.m_{t}(\mu=M) \simeq \frac{m_{1} m_{t}^{\prime}}{M}\right|_{\mu=M},
\end{gathered}
$$

and

$$
\tilde{x}_{2}(\mu=M-0) \simeq \tilde{x}_{2}(\mu=M+0)+\left.\frac{m_{t}^{\prime 2}}{M^{2}}\right|_{\mu=M},
$$

at the tree level. Higher order corrections to Eqs. (III.29) and (III.30) are suppressed by $1 /(4 \pi)^{2}$ or $m_{1}^{2} / M^{2}$ and thus irrelevant in the evaluation of $\alpha S$ and $\alpha T$ at the $\mathcal{O}\left(10^{-3}\right)$ level.

Note that the operator $x_{1}$ affects the $W$ and $Z$ couplings with light fermions. In order to guarantee universal $W$ and $Z$ couplings with light quarks and leptons, we 
thus need to arrange $m_{1} / M$ universal to fermion flavors in Eq.(III.29). As we stressed in the previous subsection, the same renormalization group equation Eq.(III.22) is applied for the ratio $m_{1} / M$ to all fermion flavors other than the third generation quarks. The universality of the weak gauge boson couplings is therefore ensured once we assume universal $m_{1}$ and $M$ at the cutoff scale. The weak gauge boson couplings with the third generation quarks such as the $Z b \bar{b}$ vertex, on the other hand, may be affected by the effects coming from the renormalization group equation Eq.(III.17). We will study this effect in a separated publication.

Loop level corrections to the matching conditions of $f_{1,2}^{2}$ and $\alpha_{(i) j}$ are also suppressed by $1 /(4 \pi)^{2}$, which can be safely neglected in the evaluation of $\alpha S$ and $\alpha T$. It is easy to see that the matching conditions of $f_{1,2}^{2}$ and $\alpha_{(i) j}$ are trivial at the tree level.

The matching condition of $\beta_{(2)}$ needs to be treated more carefully. The one-loop corrected matching condition of $\beta_{(2)}$ is

$$
\left.\beta_{(2)} f_{2}^{2}\right|_{\mu=M-0}=\left.\beta_{(2)} f_{2}^{2}\right|_{\mu=M+0}+\left.\frac{N_{c}}{24 \pi^{2}} \frac{m_{t}^{\prime 4}}{M^{2}}\right|_{\mu=M} .
$$

The non-trivial correction arises from the KK top-quark loop diagram [56]. As we will see later, the large value of $m_{t}^{\prime}$ makes the one loop correction

$$
\frac{N_{c}}{24 \pi^{2}} \frac{m_{t}^{\prime 4}}{M^{2}}
$$

important in the evaluation of $\alpha T$ at the $\mathcal{O}\left(10^{-3}\right)$ level.

\section{Below KK fermion mass $M$}

Below the KK fermion mass scale $M$, the third generation quark fields couple with $U_{1} U_{2}$ through Eq.(II.16), which causes non-trivial running of $f_{12}^{2}$ as

$$
\mu \frac{d}{d \mu} f_{12}^{2}=-\frac{N_{c}}{4 \pi^{2}} m_{t}^{2},
$$

where we have neglected terms proportional to $f_{12}^{2}$ in the RHS of Eq.(III.34). This is justified so long as $f_{12}^{2} \ll m_{t}^{2}$. We also find

$$
\mu \frac{d}{d \mu} x_{1}^{\prime}=-\frac{2}{3(4 \pi)^{2}} \frac{x_{1}}{f_{1}^{2}} .
$$

See Appendix A for a derivation of Eq. III.35).

The renormalization group equations of other $\mathcal{O}\left(p^{2}\right)$ coefficients are

$$
\begin{aligned}
\mu \frac{d}{d \mu} f_{1}^{2} & =\frac{3}{(4 \pi)^{2}}\left(g_{0}^{2}+g_{1}^{2}\right) f_{1}^{2}, \\
\mu \frac{d}{d \mu} f_{2}^{2} & =\frac{3}{(4 \pi)^{2}}\left(g_{1}^{2}+\frac{1}{2} g_{2}^{2}\right) f_{2}^{2}, \\
\mu \frac{d}{d \mu}\left(\beta_{(2)} f_{2}^{2}\right) & =\frac{3}{4(4 \pi)^{2}} g_{2}^{2} f_{2}^{2} .
\end{aligned}
$$

We also find the renormalization group equations for the gauge coupling strengths,

$$
\begin{aligned}
\mu \frac{d}{d \mu}\left(\frac{1}{g_{0}^{2}}\right) & =\frac{1}{(4 \pi)^{2}}\left[\frac{44}{3}-\frac{1}{6}-\frac{2}{3} N_{g}\left(N_{c}+1\right)\right], \\
\mu \frac{d}{d \mu}\left(\frac{1}{g_{1}^{2}}\right) & =\frac{1}{(4 \pi)^{2}}\left[\frac{44}{3}-\frac{2}{6}\right], \\
\mu \frac{d}{d \mu}\left(\frac{1}{g_{2}^{2}}\right) & =\frac{1}{(4 \pi)^{2}}\left[-\frac{1}{6}-\frac{4}{3} N_{g}\left(\frac{11}{18} N_{c}+\frac{3}{2}\right)\right], \\
\mu \frac{d}{d \mu}\left(\frac{1}{g_{s}^{2}}\right) & =\frac{1}{(4 \pi)^{2}}\left[22-\frac{8}{3} N_{g}\right] .
\end{aligned}
$$

The renormalization group equations for $\alpha_{(i) j}$ are unchanged, i.e., Eqs. III.9 - III.13) are valid below $M$ as long as $f_{12}^{2} \ll f_{1}^{2}, f_{2}^{2}$. The renormalization group equation for the delocalization operator $x_{1}$ is 67 .

$$
\mu \frac{d}{d \mu} x_{1}=\frac{3 g_{1}^{2}}{(4 \pi)^{2}} x_{1} .
$$

\section{ELECTROWEAK CHIRAL LAGRANGIAN}

Below the $W^{\prime}$ mass scale, phenomenology of the three site Higgsless model can be described by the two-site model, i.e., the electroweak chiral Lagrangian [58, 59, 60, 61, 62, 63.,

$$
\begin{aligned}
\mathcal{L}_{2}= & \frac{f^{2}}{4} \operatorname{tr}\left[\left(D_{\mu} U\right)^{\dagger}\left(D^{\mu} U\right)\right]-\frac{1}{2 g_{W}^{2}} \operatorname{tr}\left[W_{\mu \nu} W^{\mu \nu}\right] \\
& -\frac{1}{2 g_{Y}^{2}} \operatorname{tr}\left[B_{\mu \nu} B^{\mu \nu}\right] .
\end{aligned}
$$

where

$$
D_{\mu} U=\partial_{\mu} U+i W_{\mu} U-i U B_{\mu}, \quad U \equiv U_{1} U_{2} .
$$

There also exists a custodial symmetry violating $\mathcal{O}\left(p^{2}\right)$ operator

$$
\mathcal{L}_{2}^{\prime}=\beta \frac{f^{2}}{4} \operatorname{tr}\left[U^{\dagger}\left(D_{\mu} U\right) \tau^{3}\right] \operatorname{tr}\left[U^{\dagger}\left(D^{\mu} U\right) \tau^{3}\right] .
$$

$\mathcal{O}\left(p^{4}\right)$ operators

$$
\begin{aligned}
\mathcal{L}_{4}= & \alpha_{1} \operatorname{tr}\left[W_{\mu \nu} U B^{\mu \nu} U^{\dagger}\right] \\
& -2 i \alpha_{2} \operatorname{tr}\left[\left(D_{\mu} U\right)^{\dagger}\left(D_{\nu} U\right) B_{\mu \nu}\right] \\
& -2 i \alpha_{3} \operatorname{tr}\left[W^{\mu \nu}\left(D_{\mu} U\right)\left(D_{\nu} U\right)^{\dagger}\right] \\
& +\alpha_{4} \operatorname{tr}\left[\left(D_{\mu} U\right)\left(D_{\nu} U\right)^{\dagger}\right] \operatorname{tr}\left[\left(D^{\mu} U\right)\left(D^{\nu} U\right)^{\dagger}\right] \\
& +\alpha_{5} \operatorname{tr}\left[\left(D_{\mu} U\right)\left(D^{\mu} U\right)^{\dagger}\right] \operatorname{tr}\left[\left(D_{\nu} U\right)\left(D^{\nu} U\right)^{\dagger}\right]
\end{aligned}
$$

are also introduced in the two-site model. 
Using the technique described in Ref. [67], we find matching conditions,

$$
\begin{aligned}
f^{2} & \simeq \frac{f_{1}^{2} f_{2}^{2}}{f_{1}^{2}+f_{2}^{2}}+f_{12}^{2} \\
\beta f^{2} & \simeq \beta_{(2)} \frac{f_{1}^{4} f_{2}^{2}}{\left(f_{1}^{2}+f_{2}^{2}\right)^{2}} \\
\frac{1}{g_{W}^{2}} & \simeq \frac{1}{g_{0}^{2}} \\
\frac{1}{g_{Y}^{2}} & \simeq \frac{1}{g_{2}^{2}}
\end{aligned}
$$

and

$$
\begin{aligned}
\alpha_{1} \simeq & -\frac{1}{g_{1}^{2}} \frac{f_{1}^{2} f_{2}^{2}}{\left(f_{1}^{2}+f_{2}^{2}\right)^{2}}+\left(\alpha_{(1) 1}+\frac{x_{1}}{g_{0}^{2}}\right) \frac{f_{2}^{2}}{f_{1}^{2}+f_{2}^{2}} \\
& +\alpha_{(2) 1} \frac{f_{1}^{2}}{f_{1}^{2}+f_{2}^{2}},
\end{aligned}
$$

at the scale

$$
\mu=M_{W^{\prime}} \simeq g_{1} \frac{\sqrt{f_{1}^{2}+f_{2}^{2}}}{2} .
$$

We next consider the renormalization group flow in the two-site model. The renormalization group equations of the two-site model are given by

$$
\begin{aligned}
\mu \frac{d}{d \mu} f^{2} & =\frac{3}{(4 \pi)^{2}}\left(g_{W}^{2}+\frac{1}{2} g_{Y}^{2}\right) f^{2}-\frac{1}{4 \pi^{2}} N_{c} m_{t}^{2}, \\
\mu \frac{d}{d \mu}\left(\beta f^{2}\right) & =\frac{3}{4(4 \pi)^{2}} g_{Y}^{2} f^{2}, \\
\mu \frac{d}{d \mu}\left(\frac{1}{g_{W}^{2}}\right) & =\frac{1}{(4 \pi)^{2}}\left[\frac{44}{3}-\frac{1}{6}-\frac{2}{3} N_{g}\left(N_{c}+1\right)\right], \\
\mu \frac{d}{d \mu}\left(\frac{1}{g_{Y}^{2}}\right) & =\frac{1}{(4 \pi)^{2}}\left[-\frac{1}{6}-\frac{4}{3} N_{g}\left(\frac{11}{18} N_{c}+\frac{3}{2}\right)\right],
\end{aligned}
$$

and

$$
\begin{aligned}
\mu \frac{d}{d \mu} \alpha_{1} & =\frac{1}{6(4 \pi)^{2}}, \\
\mu \frac{d}{d \mu} \alpha_{2} & =\frac{1}{12(4 \pi)^{2}}, \\
\mu \frac{d}{d \mu} \alpha_{3} & =\frac{1}{12(4 \pi)^{2}}, \\
\mu \frac{d}{d \mu} \alpha_{4} & =-\frac{1}{6(4 \pi)^{2}} \\
\mu \frac{d}{d \mu} \alpha_{5} & =-\frac{1}{12(4 \pi)^{2}} .
\end{aligned}
$$

\section{V. $S$ AND $T$ PARAMETERS}

We are now ready to evaluate $S$ and $T$ parameters in the three site Higgsless model. These parameters are defined as the deviations from the standard model,

$$
\begin{aligned}
\alpha S & \equiv-16 \pi \alpha\left[\alpha_{1}(\mu)-\alpha_{1}^{\mathrm{SM}}(\mu)\right], \\
\alpha T & \equiv 2\left[\beta(\mu)-\beta^{\mathrm{SM}}(\mu)\right],
\end{aligned}
$$

with $\alpha_{1}^{\mathrm{SM}}(\mu), \beta^{\mathrm{SM}}(\mu)$ being the low energy chiral coefficients of the "reference" standard model with a "reference" heavy Higgs boson mass $M_{H, \text { ref }}$,

$$
\begin{aligned}
\alpha_{1}^{\mathrm{SM}}(\mu) & =\frac{1}{6(4 \pi)^{2}} \ln \frac{\mu}{M_{H, \mathrm{ref}}}, \\
\beta^{\mathrm{SM}}(\mu) & =\frac{3}{4} \frac{g_{Y}^{2}}{(4 \pi)^{2}} \ln \frac{\mu}{M_{H, \mathrm{ref}}} .
\end{aligned}
$$

The running of $\alpha_{1}$ is described by Eq. (IV.15). We readily find

$$
\alpha_{1}(\mu)=\alpha_{1}\left(M_{W^{\prime}}\right)+\frac{1}{6(4 \pi)^{2}} \ln \frac{\mu}{M_{W^{\prime}}} .
$$

Combining this expression with Eq.(V.1) and Eq.(V.3), we obtain

$$
S=-16 \pi \alpha_{1}\left(M_{W^{\prime}}\right)+\frac{1}{6 \pi} \ln \frac{M_{W^{\prime}}}{M_{H, \text { ref }}} .
$$

We next turn to the $T$ parameter. Eq.(IV.12) reads

$$
\mu \frac{d}{d \mu} \beta=\frac{3}{4(4 \pi)^{2}} g_{Y}^{2}-\beta \frac{1}{f^{2}} \mu \frac{d}{d \mu} f^{2} .
$$

Note that

$$
\beta \ll 1, \quad \frac{1}{f^{2}} \mu \frac{d}{d \mu} f^{2} \ll 1 .
$$

The second term in the RHS of Eq.(V.7) can thus be neglected safely. We then obtain

$$
\beta(\mu)=\beta\left(M_{W^{\prime}}\right)+\frac{3}{4} \frac{g_{Y}^{2}}{(4 \pi)^{2}} \ln \frac{\mu}{M_{W^{\prime}}},
$$

and thus

$$
T=\frac{2}{\alpha} \beta\left(\mu=M_{W^{\prime}}\right)-\frac{3}{8 \pi c^{2}} \ln \frac{M_{W^{\prime}}}{M_{H, \text { ref }}} .
$$

We next use the matching conditions Eq. (IV.6) and Eq.(IV.9), and obtain

$$
\begin{aligned}
S= & \left.\frac{16 \pi \kappa}{(1+\kappa)^{2}}\left[\frac{1}{g_{1}^{2}}-\frac{x_{1}}{g_{0}^{2}}(1+\kappa)\right]\right|_{\mu=M_{W^{\prime}}}+\frac{1}{6 \pi} \ln \frac{M_{W^{\prime}}}{M_{H, \mathrm{ref}}}, \\
& -\left.\frac{16 \pi}{1+\kappa}\left(\kappa \alpha_{(1) 1}+\alpha_{(2) 1}\right)\right|_{\mu=M_{W^{\prime}}}+(\mathrm{V} .11) \\
T= & \left.\frac{1}{\alpha} \frac{2}{1+\kappa} \beta_{(2)}\right|_{\mu=M_{W^{\prime}}}-\frac{3}{8 \pi c^{2}} \ln \frac{M_{W^{\prime}}}{M_{H, \mathrm{ref}}}, \quad(\mathrm{V} .12)
\end{aligned}
$$


with $\kappa$ being defined as

$$
\left.\kappa \equiv \frac{f_{2}^{2}}{f_{1}^{2}}\right|_{\mu=M_{W^{\prime}}} .
$$

Assuming $f_{12}^{2} \ll f_{1}^{2}, f_{2}^{2}$, Eq. (IV.5 leads

$$
\frac{1}{f_{1}^{2}} \simeq \frac{\kappa}{1+\kappa} \frac{1}{f^{2}}, \quad \frac{1}{f_{2}^{2}} \simeq \frac{1}{1+\kappa} \frac{1}{f^{2}} .
$$

In Eqs.(V.11) and (V.12), $g_{0}, g_{1}, x_{1}, \alpha_{(1) 1}$ and $\alpha_{(2) 1}$ are renormalized at the scale

$$
\mu=M_{W^{\prime}}=g_{1} \frac{\sqrt{f_{1}^{2}+f_{2}^{2}}}{2} \simeq g_{1} f \frac{1+\kappa}{2 \sqrt{\kappa}},
$$

with $f$ being the electroweak scale $f \simeq 250 \mathrm{GeV}$.

It is possible to express these $S$ and $T$ formulas in terms of the parameters renormalized at the KK fermion mass scale $M$. Using the renormalization group equations given in Section III.C, we obtain

$$
\begin{aligned}
S= & \left.\frac{16 \pi \kappa}{(1+\kappa)^{2}}\left[\frac{1}{g_{1}^{2}}-\frac{x_{1}}{g_{0}^{2}}(1+\kappa)\right]\right|_{\mu=M} \\
& -\left.\frac{16 \pi}{1+\kappa}\left(\kappa \alpha_{(1) 1}+\alpha_{(2) 1}\right)\right|_{\mu=M}+\frac{1}{6 \pi} \ln \frac{M_{W^{\prime}}}{M_{H, \mathrm{ref}}} \\
& -\frac{1}{6 \pi} \ln \frac{M_{W^{\prime}}}{M}+\frac{43 \kappa}{3(1+\kappa)^{2} \pi} \ln \frac{M_{W^{\prime}}}{M} \\
& -\frac{3 \kappa}{(1+\kappa) \pi} \frac{g_{1}^{2}}{g_{0}^{2}} x_{1} \ln \frac{M_{W^{\prime}}}{M}, \\
T= & \left.\frac{1}{\alpha} \frac{2}{1+\kappa} \beta_{(2)}\right|_{\mu=M-0}-\frac{3}{8 \pi c^{2}} \ln \frac{M_{W^{\prime}}}{M_{H, \mathrm{ref}}} \\
& +\frac{3}{8 \pi(1+\kappa) c^{2}} \ln \frac{M_{W^{\prime}}}{M},
\end{aligned}
$$

at the one loop level. We have neglected running of the weak gauge coupling constant $g_{0}$ in these formulas. Taking $\kappa=1$, we find

$$
\begin{aligned}
S= & \left.4 \pi\left[\frac{1}{g_{1}^{2}}-2 \frac{x_{1}}{g_{0}^{2}}\right]\right|_{\mu=M} \\
& -\left.8 \pi\left(\alpha_{(1) 1}+\alpha_{(2) 1}\right)\right|_{\mu=M}+\frac{1}{6 \pi} \ln \frac{M_{W^{\prime}}}{M_{H, \mathrm{ref}}} \\
& +\frac{41}{12 \pi} \ln \frac{M_{W^{\prime}}}{M} \\
& -\frac{3}{2 \pi} \frac{g_{1}^{2}}{g_{0}^{2}} x_{1} \ln \frac{M_{W^{\prime}}}{M}, \\
T= & \left.\frac{1}{\alpha} \beta_{(2)}\right|_{\mu=M-0}-\frac{3}{8 \pi c^{2}} \ln \frac{M_{W^{\prime}}}{M_{H, \mathrm{ref}}} \\
& +\frac{3}{16 \pi c^{2}} \ln \frac{M_{W^{\prime}}}{M} .
\end{aligned}
$$

We now compare Eqs.(V.18) and (V.19) with those given in Refs. 66, 67]. Note that the effective theory used in Refs. [66, 67] does not include the KK fermion. This theory is therefore valid only below the KK fermion mass scale $M$. The cutoff scale used in Refs. [66, 67] should then be regarded as the KK fermion mass scale $M$. It is easy to see that Eqs.( (V.18) and (V.19) correspond exactly to those found in Refs. [66, 67].

Note that Eqs.(V.18) and (V.19) are written in terms of the parameters renormalized at the KK fermion mass scale. These parameters are not directly related with the on-shell observables, however. In order to perform a more sensible phenomenological fit, we next express $S$ and $T$ in terms of on-shell observables.

We first consider the $S$ parameter. In order to determine the value $x_{1}\left(\mu=M_{W^{\prime}}\right)$ in Eq.(V.11), we use

$$
\begin{gathered}
g_{W^{\prime} f f}=\left.\frac{g_{0}^{2} g_{1}}{1+\kappa}\left[-\frac{1}{g_{1}^{2}}+\frac{x_{1}}{g_{0}^{2}}(1+\kappa)\right]\right|_{\mu=M_{W^{\prime}}} \\
+g_{1} x_{1}^{\prime}\left(M_{W^{\prime}}\right) M_{W^{\prime}}^{2}
\end{gathered}
$$

with $g_{W^{\prime} f f}$ being the on-shell $W^{\prime}$ couplings with massless quarks and leptons. Here $x_{1}^{\prime}$ is defined in Eq. (II.20). From Eq.(II.22) and Eq.(III.35), we see that $x_{1}^{\prime}$ can be written in terms of $x_{1}$,

$$
x_{1}^{\prime}\left(\mu=M_{W^{\prime}}\right)=-\frac{1}{3(4 \pi)^{2}} \frac{x_{1}}{f_{1}^{2}} \ln \frac{M_{W^{\prime}}^{2}}{M^{2}} .
$$

Note here that the operator $x_{1}^{\prime}$ makes non-trivial correction to the on-shell $g_{W^{\prime} f f}$ couplings. Due to the large value of $M_{W^{\prime}}$, the effect from $x_{1}^{\prime}$ turns out to be numerically significant.

Note that the delocalization coefficient $x_{1}$ can always be tuned 49, 71] so as to minimize the $S$ parameter in Eq.(V.11). Phenomenologically we know

$$
S \lesssim 0.1
$$

and thus

$$
\left.x_{1}\left(M_{W^{\prime}}\right) \simeq \frac{1}{1+\kappa} \frac{g_{0}^{2}}{g_{1}^{2}}\right|_{\mu=M_{W^{\prime}}} .
$$

This allows us to expand $x_{1}$ around Eq.(V.23). Eq.(V.20) then gives

$$
\begin{aligned}
\left.\frac{x_{1}}{g_{0}^{2}}\right|_{\mu=M_{W^{\prime}}}= & \left.\frac{1}{1+\kappa} \frac{1}{g_{1}^{2}}\right|_{\mu=M_{W^{\prime}}}+\frac{g_{W^{\prime} f f}}{g_{0}^{2} g_{1}} \\
& +\frac{\kappa}{3(1+\kappa)^{2}(4 \pi)^{2}} \ln \frac{M_{W^{\prime}}^{2}}{M^{2}}+\cdots .
\end{aligned}
$$

Combining Eqs.(V.11), (V.21) and (V.24), we obtain

$$
\begin{aligned}
S= & -4 \sqrt{\kappa} \frac{s^{2}}{\alpha} \frac{M_{W}}{M_{W^{\prime}}} \frac{g_{W^{\prime} f f}}{g_{W}} \\
& -\left.\frac{16 \pi}{1+\kappa}\left(\kappa \alpha_{(1) 1}+\alpha_{(2) 1}\right)\right|_{\mu=M_{W^{\prime}}} \\
& -\frac{\kappa^{2}}{(1+\kappa)^{3}} \frac{1}{3 \pi} \ln \frac{M_{W^{\prime}}^{2}}{M^{2}}+\frac{1}{12 \pi} \ln \frac{M_{W^{\prime}}^{2}}{M_{H, \mathrm{ref}}^{2}},
\end{aligned}
$$


with

$$
s^{2} \equiv \frac{e^{2}}{g_{W}^{2}}
$$

Here we neglected $(4 \pi)^{-2} g_{W^{\prime} f f} \ln \left(M_{W} / M_{W^{\prime}}\right)$ corrections. We also used the tree level formula

$$
\frac{g_{0}}{g_{1}}=\frac{1+\kappa}{\sqrt{\kappa}} \frac{M_{W}}{M_{W^{\prime}}} .
$$

We next use the renormalization group equations Eq.(III.9) in order to evaluate $\left.\left(\kappa \alpha_{(1) 1}+\alpha_{(2) 1}\right)\right|_{\mu=M_{W^{\prime}}}$ in Eq.(V.25). We obtain

$$
\begin{aligned}
S= & -4 \sqrt{\kappa} \frac{s^{2}}{\alpha} \frac{M_{W}}{M_{W^{\prime}}} \frac{g_{W^{\prime} f f}}{g_{W}} \\
& -\left.\frac{16 \pi}{1+\kappa}\left(\kappa \alpha_{(1) 1}+\alpha_{(2) 1}\right)\right|_{\mu=\Lambda} \\
& -\frac{\kappa^{2}}{(1+\kappa)^{3}} \frac{1}{3 \pi} \ln \frac{M_{W^{\prime}}^{2}}{M^{2}}+\frac{1}{12 \pi} \ln \frac{\Lambda^{2}}{M_{H, \mathrm{ref}}^{2}} .
\end{aligned}
$$

We next turn to the $T$ parameter formula Eq. (V.12). Using the renormalization group equations Eqs.(III.3), (III.38) and the matching condition Eq.(III.32), we find $\beta_{(2)}\left(M_{W^{\prime}}\right)$ in Eq.(V.12) can be expressed as

$\beta_{(2)}\left(M_{W^{\prime}}\right)=\beta_{(2)}(\Lambda)+\frac{3 g_{2}^{2}}{8(4 \pi)^{2}} \ln \frac{M_{W^{\prime}}^{2}}{\Lambda^{2}}+\left.\frac{N_{c}}{24 \pi^{2}} \frac{1}{f_{2}^{2}} \frac{m_{t}^{\prime 4}}{M^{2}}\right|_{\mu=M}$.

Combining Eq.(III.29) and Eq.(III.30), we see

$$
\left.\frac{m_{t}^{\prime 4}}{M^{2}}\right|_{\mu=M}=\left.\frac{m_{t}^{4}}{M^{2}} \frac{1}{x_{1}^{2}}\right|_{\mu=M} .
$$

For the one loop evaluation of $\beta_{(2)}$, it is enough to evaluate $x_{1}$ and $m_{t}$ at tree level. We use the value of $x_{1}$ renormalized at the $M_{W^{\prime}}$ scale

$$
x_{1}(M) \simeq x_{1}\left(M_{W^{\prime}}\right) .
$$

We thus find

$$
\left.\frac{m_{t}^{\prime 4}}{M^{2}}\right|_{\mu=M} \simeq(1+\kappa)^{2} \frac{g_{1}^{4}}{g_{0}^{4}} \frac{m_{t}^{4}}{M^{2}}\left[1+(1+\kappa) \frac{g_{1}}{g_{0}} \frac{g_{W^{\prime} f f}}{g_{0}}\right]^{-2},
$$

where we used the first two leading terms in Eq.(V.24) for the evaluation of $x_{1}$. Putting Eq.(V.27) into Eq.(V.32) we see

$$
\left.\frac{m_{t}^{\prime 4}}{M^{2}}\right|_{\mu=M} \simeq \frac{\kappa^{2}}{(1+\kappa)^{2}} \frac{M_{W^{\prime}}^{4}}{M_{W}^{4}} \frac{m_{t}^{4}}{M^{2}}\left[1+\sqrt{\kappa} \frac{M_{W^{\prime}}}{M_{W}} \frac{g_{W^{\prime} f f}}{g_{W}}\right]^{-2},
$$

and thus

$$
\begin{aligned}
\beta_{(2)}\left(M_{W^{\prime}}\right) \simeq & \beta_{(2)}(\Lambda)+\frac{3 \alpha}{32 \pi c^{2}} \ln \frac{M_{W^{\prime}}^{2}}{\Lambda^{2}} \\
+ & \frac{N_{c}}{24 \pi^{2}} \frac{\kappa^{2}}{(1+\kappa)^{3}} \frac{1}{f^{2}} \frac{M_{W^{\prime}}^{4}}{M_{W}^{4}} \frac{m_{t}^{4}}{M^{2}} \times \\
& \times\left[1+\sqrt{\kappa} \frac{M_{W^{\prime}}}{M_{W}} \frac{g_{W^{\prime} f f}}{g_{W}}\right]^{-2} .
\end{aligned}
$$

Putting Eq.(V.34) into Eq.(V.12), we reach an expression for $T$,

$$
\begin{aligned}
T= & \frac{2}{\alpha} \frac{1}{1+\kappa} \beta_{(2)}(\Lambda)-\frac{1}{1+\kappa} \frac{3}{16 \pi c^{2}} \ln \frac{\Lambda^{2}}{M_{W^{\prime}}^{2}} \\
& -\frac{3}{16 \pi c^{2}} \ln \frac{M_{W^{\prime}}^{2}}{M_{H, \mathrm{ref}}^{2}} \\
& +\frac{N_{c}}{12 \pi^{2} \alpha} \frac{\kappa^{2}}{(1+\kappa)^{4}} \frac{1}{f^{2}} \frac{M_{W^{\prime}}^{4}}{M_{W}^{4}} \frac{m_{t}^{4}}{M^{2}} \times \\
& \times\left[1+\sqrt{\kappa} \frac{M_{W^{\prime}}}{M_{W}} \frac{g_{W^{\prime} f f}}{g_{W}}\right]^{-2} .
\end{aligned}
$$

\section{COMPARISON WITH ELECTROWEAK FIT}

Now we have formulas both for $S$ and $T$ parameters

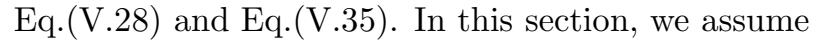

$$
\kappa=1
$$

and

$$
\alpha_{(1) 1}(\Lambda)=\alpha_{(2) 1}(\Lambda)=0, \quad \beta_{(2)}(\Lambda)=0,
$$

in Eq. (V.28) and Eq.(V.35). The assumption Eq.(VI.1) maximizes the scale of unitarity violation. Eq.(VI.2) is justified if the physics above the cutoff scale $\Lambda$ does not affect much to these oblique parameters $S$ and $T$. These assumptions lead to

$$
\begin{aligned}
S= & -4 \frac{s^{2}}{\alpha} \frac{M_{W}}{M_{W^{\prime}}} \frac{g_{W^{\prime} f f}}{g_{W}} \\
& -\frac{1}{24 \pi} \ln \frac{M_{W^{\prime}}^{2}}{M^{2}}+\frac{1}{12 \pi} \ln \frac{\Lambda^{2}}{M_{H, \mathrm{ref}}^{2}}, \\
T= & -\frac{3}{32 \pi c^{2}} \ln \frac{\Lambda^{2}}{M_{W^{\prime}}^{2}}-\frac{3}{16 \pi c^{2}} \ln \frac{M_{W^{\prime}}^{2}}{M_{H, \mathrm{ref}}^{2}} \\
& +\frac{N_{c}}{192 \pi s^{2}} \frac{M_{W^{\prime}}^{4}}{M_{W}^{6}} \frac{m_{t}^{4}}{M^{2}}\left[1+\frac{M_{W^{\prime}}}{M_{W}} \frac{g_{W^{\prime} f f}}{g_{W}}\right]^{-2} .
\end{aligned}
$$

Note that these expressions are written in terms of physical observables, such as $M_{W^{\prime}}, g_{W^{\prime} f f}$ and the KK fermion mass $M$, except for the cutoff scale $\Lambda$. Note also that the cutoff scale $\Lambda$ should be below the naive dimensional analysis (NDA) [72] scale $4 \pi f_{1}=4 \pi f_{2} \simeq 4.3 \mathrm{TeV}$. 


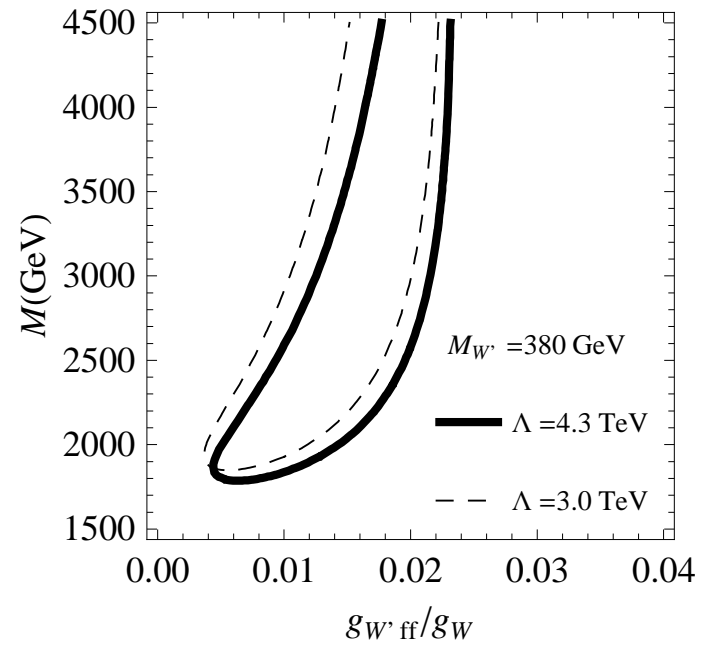

FIG. 2: Phenomenologically acceptable area in the $\left(g_{W^{\prime} f f} / g_{W}, M\right)$ plane. $M_{W^{\prime}}=380 \mathrm{GeV}$ is assumed. The region surrounded by the solid (dashed) curve satisfies the $95 \%$ CL constraint for cutoff $\Lambda=4.3 \mathrm{TeV}(3.0 \mathrm{TeV})$.

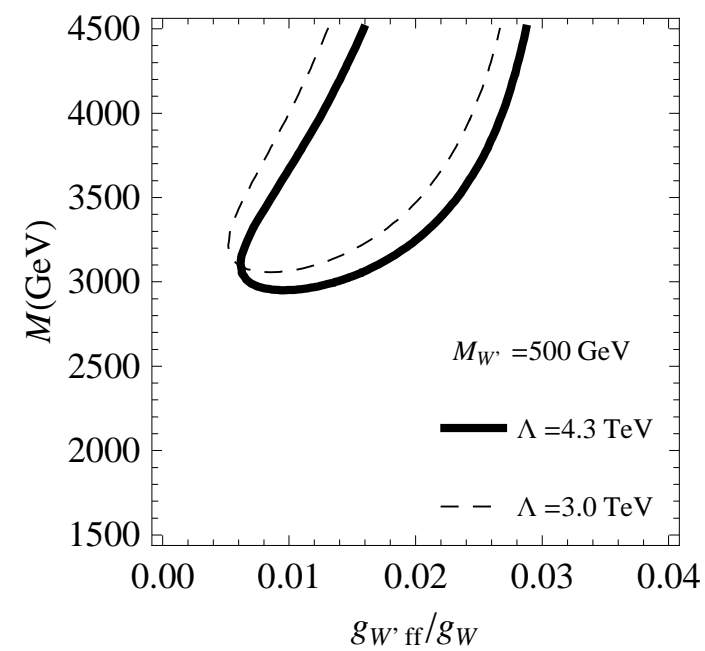

FIG. 3: Phenomenologically acceptable area in the $\left(g_{W^{\prime} f f} / g_{W}, M\right)$ plane. $M_{W^{\prime}}=500 \mathrm{GeV}$ is assumed. The region surrounded by the solid (dashed) curve satisfies the $95 \%$ CL constraint for cutoff $\Lambda=4.3 \mathrm{TeV}(3.0 \mathrm{TeV})$.

We compare these results with values of $S$ and $T$ extracted from the precision electroweak fit,

$$
\begin{aligned}
& S=-0.21 \pm 0.09, \\
& T=0.21 \pm 0.09 .
\end{aligned}
$$

The central values of Eqs.(VI.5), (VI.6) are for a "reference" Higgs boson mass $M_{H \text {,ref }}=1 \mathrm{TeV}$, and are taken from Figure 10.4 in Ref. [75]. Note that there exists strong error correlation between $S$ and $T$,

$$
\rho_{S T}=0.84 \text {. }
$$

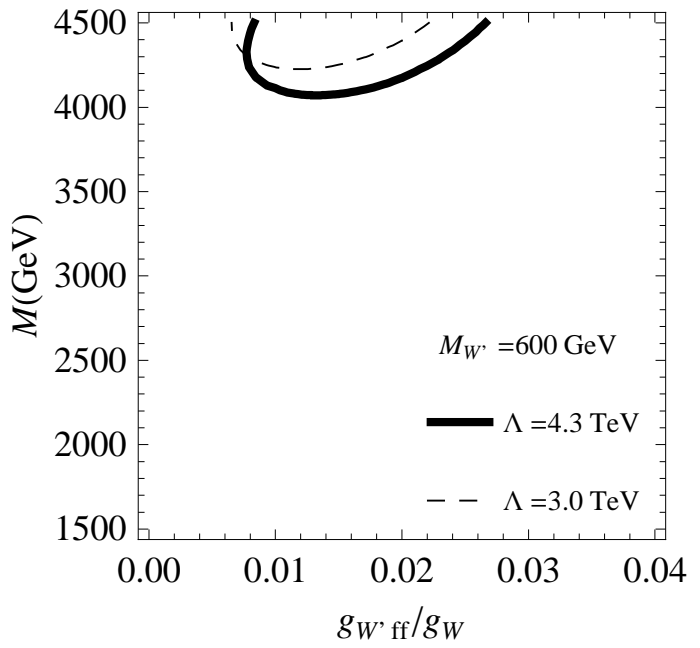

FIG. 4: Phenomenologically acceptable area in the $\left(g_{W^{\prime} f f} / g_{W}, M\right)$ plane. $M_{W^{\prime}}=600 \mathrm{GeV}$ is assumed. The region surrounded by the solid (dashed) curve satisfies the $95 \%$ CL constraint for cutoff $\Lambda=4.3 \mathrm{TeV}(3.0 \mathrm{TeV})$.

The $95 \%$ CL allowed region in the $(S, T)$ plane is then given by

$$
\begin{aligned}
\frac{\left(S-S_{0}\right)^{2}}{\sigma_{S}^{2}} & +\frac{\left(T-T_{0}\right)^{2}}{\sigma_{T}^{2}}-2 \rho_{S T} \frac{S-S_{0}}{\sigma_{S}} \frac{T-T_{0}}{\sigma_{T}} \\
& <5.99 \times\left(1-\rho_{S T}^{2}\right),
\end{aligned}
$$

with $S_{0}=-0.21, T_{0}=0.21, \sigma_{S}=0.09, \sigma_{T}=0.09$. For fixed $M_{W^{\prime}}$ and $\Lambda$, we now obtain bounds on $g_{W^{\prime} f f}$ and $M$ from the constraint in the $(S, T)$ plane. The allowed regions satisfying the $95 \% \mathrm{CL}$ constraint Eq. (VI.8) are depicted in Figure 2 in the $\left(g_{W^{\prime} f f} / g_{W}, M\right)$ plane for $M_{W^{\prime}}=380 \mathrm{GeV}$. In the plot we used $\alpha^{-1}=128.91, G_{F}=$ $1 /\left(\sqrt{2} f^{2}\right)=1.16637 \times 10^{-5} \mathrm{GeV}^{-2}, s^{2}=1-c^{2}=0.231$, and $m_{t}=174.2 \mathrm{GeV}$.

The region surrounded by the solid (dashed) curve satisfies the 95\% CL constraint Eq.(VI.8) for cutoff $\Lambda=$ $4.3 \mathrm{TeV}(3.0 \mathrm{TeV})$. We emphasize that the $W^{\prime}$ boson needs to have non-zero $g_{W^{\prime} f f}$ in order to satisfy the precision electroweak constraints. This is in sharp contrast to the tree level analysis [56], where the ideal delocalization [51] implies completely fermiophobic $W^{\prime}$. The KK fermion mass is also severely constrained. We find $M \gtrsim 1.8 \mathrm{TeV}$ for $M_{W^{\prime}}=380 \mathrm{GeV}$ and $\Lambda=4.3 \mathrm{TeV}$. The change of the cutoff assumption to $\Lambda=3.0 \mathrm{TeV}$ affects little in Figure 2. The dependence on the cutoff assumption is not significant in this numerical analysis.

Figures 3 and 4 show similar plots for $M_{W^{\prime}}=500 \mathrm{GeV}$ and for $600 \mathrm{GeV}$. We see the KK fermion lower mass bound becomes much severer for heavier $M_{W^{\prime}}$. This severe constraint on $M$ essentially comes from the $T$ parameter constraint through the KK top-quark loop effect [56]. Figure 5 shows the bound on the KK fermion mass $M$ as functions of $M_{W^{\prime}}$. Here $g_{W^{\prime} f f}$ is tuned so as 
to maximize the likelihood in the fit. Again, we find the cutoff dependence is rather small.

We now turn to the bounds on $M_{W^{\prime}}$. As shown in Ref.[56], the LEP-II triple gauge vertex bound gives a lower bound on the $W^{\prime}$ mass

$$
M_{W^{\prime}}>380 \mathrm{GeV}
$$

As depicted in Figure [5] the lower bound on the KK fermion mass $M$ increases monotonically for increasing $M_{W^{\prime}}$. In order to keep our effective theory analysis sensible, we need to require

$$
M<\Lambda
$$

The requirement Eq. (VI.10), combined with Figure 5. leads to upper bounds on the $W^{\prime}$ boson mass

$$
M_{W^{\prime}}<610 \mathrm{GeV}
$$

for $\Lambda=4.3 \mathrm{TeV}$, and

$$
M_{W^{\prime}}<490 \mathrm{GeV}
$$

for $\Lambda=3.0 \mathrm{TeV}$.

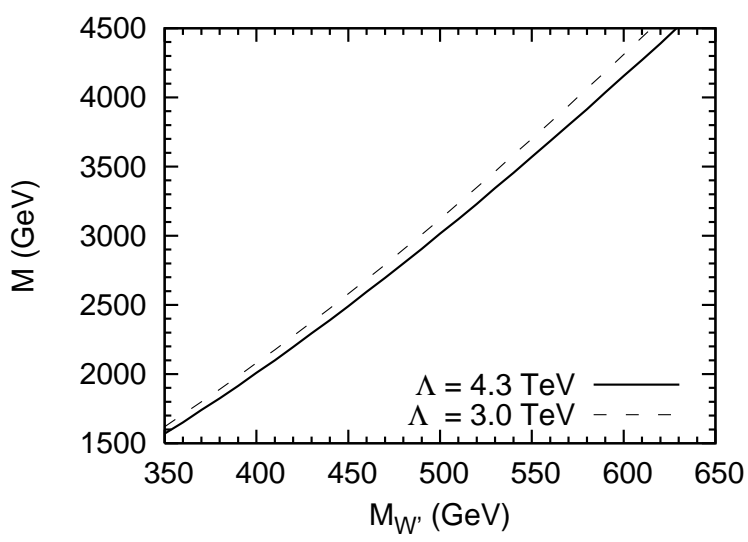

FIG. 5: Phenomenologically acceptable values of $M_{W^{\prime}}$ and $M$. The area above the curve satisfies $95 \%$ CL constraint in the $(S, T)$ plane. The $W^{\prime} f f$ coupling $g_{W^{\prime} f f}$ is assumed to be tuned so as to maximize the likelihood. The solid curve is for $\Lambda=4.3 \mathrm{TeV}$, and dashed for $\Lambda=3.0 \mathrm{TeV}$.

\section{CONCLUSIONS}

Does the three site Higgsless model survive the electroweak precision tests at loop? In this paper, we have positively answered to this question, explicitly showing that there exists a parameter region in which both $S$ and $T$ are consistent with the present bounds. We have obtained compact formulas for $S$ and $T$ at the one loop level, written in terms of physical observables such as the KK gauge boson mass $M_{W^{\prime}}$, the KK fermion mass $M$, and the $W^{\prime}$ couplings with light quarks and leptons
$g_{W^{\prime} f f}$. It has been shown, however, that the $W^{\prime} f f$ couplings and the KK fermion mass $M$ are severely constrained. Especially, unlike the tree level analysis on the ideally delocalized fermion, it has been shown that perfectly fermiophobic $W^{\prime}$ is inconsistent with the electroweak fit. Small but non-zero value of $g_{W^{\prime} f f}$ is required.

So far, collider phenomenology of Higgsless $W^{\prime}$ bosons has been analyzed assuming perfectly fermiophobic $W^{\prime}[73,74] .^{2}$ We found small $g_{W^{\prime} f f}$ of order $1 \times 10^{-2}$ $-3 \times 10^{-2}$ of $g_{W}$ is required to be consistent with the precision electroweak data. Note that such a $W^{\prime}$ boson is still effectively fermiophobic at the Tevatron collider. Actually, the production cross section $\sigma\left(q \bar{q} \rightarrow W^{\prime}\right)$ is suppressed $1 \times 10^{-4}-1 \times 10^{-3}$ compared with a $W^{\prime}$ boson having the standard model coupling strength. The existing direct search limit on $W^{\prime}$ from the Tevatron 79 cannot be applied for such an effectively fermiophobic $W^{\prime}$. Nevertheless, it should be interesting to investigate the LHC sensitivity toward such a small $g_{W^{\prime} f f}$ coupling.

\section{Acknowledgments}

We thank R. Sekhar Chivukula for his thoughtful suggestions in the preparation stage of this manuscript. T.A. thanks members of particle theory groups at Nagoya University and Tohoku University for their hospitality during the completion of this work. T.A. is supported in part by the JSPS Research Fellowships for Young Scientists. S.M. is supported by the U.S. Department of Energy under Grants No. DE-FG02-06ER41418. M.T.'s work is supported in part by the JSPS Grant-in-Aid for Scientific Research No. 20540263.

\section{APPENDIX A: DERIVATION OF EQ.(III.35)}

The gauge field $V_{0}^{\mu}$ interacts with the non-linear sigma model field $U_{1}$. Extracting the interaction term from the lowest order chiral Lagrangian, we find

$$
\left.\frac{f_{1}^{2}}{4} \operatorname{tr}\left[\left(D_{\mu} U_{1}\right)^{\dagger}\left(D^{\mu} U_{1}\right)\right]\right|_{V_{0}^{\mu}}=-\frac{f_{1}^{2}}{4} V_{0}^{a \mu} \operatorname{tr}\left[\tau^{a} i\left(\partial_{\mu} U_{1}\right)^{\dagger} U_{1}\right] .
$$

The delocalization operator Eq.(II.17) leads to the Nambu-Goldstone boson interaction with the fermion currents as

$$
\begin{aligned}
& -\sum_{n} \frac{x_{1}}{2} \bar{q}_{L}^{n, 0} \tau^{a} \gamma^{\mu} q_{L}^{n, 0} \operatorname{tr}\left[\tau^{a} i\left(\partial_{\mu} U_{1}\right)^{\dagger} U_{1}\right] \\
& -\sum_{n} \frac{x_{1}}{2} \bar{\ell}_{L}^{n, 0} \tau^{a} \gamma^{\mu} \ell_{L}^{n, 0} \operatorname{tr}\left[\tau^{a} i\left(\partial_{\mu} U_{1}\right)^{\dagger} U_{1}\right]
\end{aligned}
$$

\footnotetext{
2 Very recently, a possibility of non-fermiophobic $W^{\prime}$ is pointed
} out in the context of four-site Higgsless models [76, 77, 78]. 
Comparing Eq.A.1 with Eq.A.2, we see the delocalization operator $x_{1}$ can be absorbed into the redefinition of $V_{0 \mu}^{a}$,

$$
\tilde{V}_{0 \mu}^{a} \equiv V_{0 \mu}^{a}+2 \frac{x_{1}}{f_{1}^{2}}\left(\sum_{n} \bar{q}_{L}^{n, 0} \gamma_{\mu} \tau^{a} q_{L}^{n, 0}+\sum_{n} \bar{\ell}_{L}^{n, 0} \gamma_{\mu} \tau^{a} \ell_{L}^{n, 0}\right)
$$

in the Nambu-Goldstone boson field interaction. In Ref. [67], the authors found that the Nambu-Goldstone boson loop gives rise to a divergence of

$$
\frac{-1}{6(4 \pi)^{2} \bar{\epsilon}} \operatorname{tr}\left[V_{0 \mu \nu} U_{1} V_{1}^{\mu \nu} U_{1}^{\dagger}\right], \quad \frac{1}{\bar{\epsilon}} \equiv \frac{\Gamma(2-d / 2)}{2(4 \pi)^{d / 2-2}} .
$$

The divergence factor in Eq. A.4 can also be read from the renormalization group coefficient in Eq.(III.9). The divergence arising from $x_{1}$ can be obtained by replacing

$$
\begin{array}{r}
V_{0 \mu \nu} \rightarrow \tilde{V}_{0 \mu \nu}=V_{0 \mu \nu}+\frac{x_{1}}{f_{1}^{2}} \sum_{n} \tau^{b}\left\{\partial_{\mu}\left(\bar{q}_{L}^{n, 0} \gamma_{\nu} \tau^{b} q_{L}^{n, 0}\right)\right. \\
\left.\left.-\partial_{\nu}\left(\bar{q}_{L}^{n, 0} \gamma_{\mu} \tau^{b} q_{L}^{n, 0}\right)+\text { (lepton part }\right)\right\},
\end{array}
$$

in Eq.A.4. Dropping the total derivative terms, we then find the $x_{1}$-proportional part of the divergence,

$$
\begin{aligned}
& \left.\frac{-1}{6(4 \pi)^{2} \bar{\epsilon}} \operatorname{tr}\left[\tilde{V}_{0 \mu \nu} U_{1} V_{1}^{\mu \nu} U_{1}^{\dagger}\right]\right|_{x_{1}} \\
= & -\frac{1}{3(4 \pi)^{2} \bar{\epsilon}} \frac{x_{1}}{f_{1}^{2}} \sum_{n} \operatorname{tr}\left[\partial_{\mu}\left(\bar{q}_{L}^{n, 0} \gamma_{\nu} \tau^{b} q_{L}^{n, 0}\right) \tau^{b} U_{1} V_{1}^{\mu \nu} U_{1}^{\dagger}\right. \\
& + \text { (lepton part) } \\
= & -\frac{2}{3(4 \pi)^{2} \bar{\epsilon}} \frac{x_{1}}{f_{1}^{2}} \sum_{n}\left\{\bar{q}_{L}^{n, 0} \gamma^{\mu} D^{\nu}\left(U_{1} V_{1 \mu \nu} U_{1}^{\dagger}\right) q_{L}^{n, 0}\right. \\
& +(\text { lepton part })\} .
\end{aligned}
$$

It is now straightforward to obtain the renormalization group equation Eq. (III.35).
[1] C. Csaki, C. Grojean, H. Murayama, L. Pilo and J. Terning, Phys. Rev. D 69, 055006 (2004).

[2] P. W. Higgs, Phys. Lett. 12, 132 (1964).

[3] R. S. Chivukula, D. A. Dicus and H. J. He, Phys. Lett. B 525, 175 (2002).

[4] R. S. Chivukula and H. J. He, Phys. Lett. B 532, 121 (2002).

[5] R. S. Chivukula, D. A. Dicus, H. J. He and S. Nandi, Phys. Lett. B 562, 109 (2003).

[6] H. J. He, Int. J. Mod. Phys. A 20 (2005) 3362 arXiv:hep-ph/0412113.

[7] K. Agashe, A. Delgado, M. J. May and R. Sundrum, JHEP 0308 (2003) 050 arXiv:hep-ph/0308036.

[8] C. Csaki, C. Grojean, L. Pilo and J. Terning, Phys. Rev. Lett. 92 (2004) 101802 arXiv:hep-ph/0308038.

[9] G. Burdman and Y. Nomura, Phys. Rev. D 69 (2004) 115013 arXiv:hep-ph/0312247.

[10] G. Cacciapaglia, C. Csaki, C. Grojean and J. Terning, Phys. Rev. D 70 (2004) 075014 arXiv:hep-ph/0401160.

[11] N. Arkani-Hamed, A. G. Cohen and H. Georgi, Phys. Rev. Lett. 86 (2001) 4757 arXiv:hep-th/0104005.

[12] C. T. Hill, S. Pokorski and J. Wang, Phys. Rev. D 64 (2001) 105005 arXiv:hep-th/0104035.

[13] R. Foadi, S. Gopalakrishna and C. Schmidt, JHEP 0403 (2004) 042 arXiv:hep-ph/0312324.

[14] J. Hirn and J. Stern, Eur. Phys. J. C 34 (2004) 447 arXiv:hep-ph/0401032.

[15] R. Casalbuoni, S. De Curtis and D. Dominici, Phys. Rev. D 70 (2004) 055010 arXiv:hep-ph/0405188.

[16] R. S. Chivukula, E. H. Simmons, H. J. He, M. Kurachi and M. Tanabashi, Phys. Rev. D 70 (2004) 075008 arXiv:hep-ph/0406077.

[17] M. Perelstein, JHEP $\mathbf{0 4 1 0} \quad$ (2004) 010 arXiv:hep-ph/0408072.
[18] H. Georgi, Phys. Rev. D $71 \quad$ (2005) 015016 arXiv:hep-ph/0408067.

[19] R. Sekhar Chivukula, E. H. Simmons, H. J. He, M. Kurachi and M. Tanabashi, Phys. Rev. D 71 (2005) 035007 arXiv:hep-ph/0410154.

[20] H. Georgi, Nucl. Phys. B 266 (1986) 274.

[21] J. M. Maldacena, Adv. Theor. Math. Phys. 2 (1998) 231 [Int. J. Theor. Phys. 38 (1999) 1113] arXiv:hep-th/9711200.

[22] S. S. Gubser, I. R. Klebanov and A. M. Polyakov, Phys. Lett. B 428 (1998) 105 arXiv:hep-th/9802109.

[23] E. Witten, Adv. Theor. Math. Phys. 2 (1998) 253 arXiv:hep-th/9802150.

[24] O. Aharony, S. S. Gubser, J. M. Maldacena, H. Ooguri and Y. Oz, Phys. Rept. 323 (2000) 183 arXiv:hep-th/9905111.

[25] S. Weinberg, Phys. Rev. D 19 (1979) 1277.

[26] L. Susskind, Phys. Rev. D 20 (1979) 2619.

[27] B. Holdom, Phys. Rev. D 24 (1981) 1441.

[28] B. Holdom, Phys. Lett. B 150 (1985) 301.

[29] K. Yamawaki, M. Bando and K. i. Matumoto, Phys. Rev. Lett. 56 (1986) 1335.

[30] T. W. Appelquist, D. Karabali and L. C. R. Wijewardhana, Phys. Rev. Lett. 57 (1986) 957.

[31] T. Appelquist and L. C. R. Wijewardhana, Phys. Rev. D 35 (1987) 774.

[32] T. Appelquist and L. C. R. Wijewardhana, Phys. Rev. D 36 (1987) 568.

[33] M. Bando, T. Kugo, S. Uehara, K. Yamawaki and T. Yanagida, Phys. Rev. Lett. 54 (1985) 1215.

[34] M. Bando, T. Kugo and K. Yamawaki, Nucl. Phys. B 259 (1985) 493.

[35] M. Bando, T. Fujiwara and K. Yamawaki, Prog. Theor. Phys. 79 (1988) 1140. 
[36] M. Bando, T. Kugo and K. Yamawaki, Phys. Rept. 164 (1988) 217.

[37] M. Tanabashi, Phys. Lett. $\quad$ B $316 \quad$ (1993) 534 arXiv:hep-ph/9306237.

[38] M. Harada and K. Yamawaki, Phys. Rept. 381 (2003) 1 arXiv:hep-ph/0302103.

[39] H. Georgi, Nucl. Phys. B 331 (1990) 311.

[40] R. Casalbuoni, S. De Curtis, D. Dominici and R. Gatto, Phys. Lett. B 155 (1985) 95.

[41] R. Casalbuoni, A. Deandrea, S. De Curtis, D. Dominici, R. Gatto and M. Grazzini, Phys. Rev. D 53 (1996) 5201 arXiv:hep-ph/9510431.

[42] M. E. Peskin and T. Takeuchi, Phys. Rev. D 46 (1992) 381.

[43] G. Altarelli and R. Barbieri, Phys. Lett. B 253 (1991) 161.

[44] G. Altarelli, R. Barbieri and S. Jadach, Nucl. Phys. B 369 (1992) 3 [Erratum-ibid. B 376 (1992) 444].

[45] G. Cacciapaglia, C. Csaki, C. Grojean and J. Terning, Phys. Rev. D 71 (2005) 035015 arXiv:hep-ph/0409126.

[46] G. Cacciapaglia, C. Csaki, C. Grojean, M. Reece and J. Terning, Phys. Rev. D 72 (2005) 095018 arXiv:hep-ph/0505001.

[47] R. Foadi, S. Gopalakrishna and C. Schmidt, Phys. Lett. B 606 (2005) 157 arXiv:hep-ph/0409266.

[48] R. Foadi and C. Schmidt, Phys. Rev. D 73 (2006) 075011 arXiv:hep-ph/0509071.

[49] R. S. Chivukula, E. H. Simmons, H. J. He, M. Kurachi and M. Tanabashi, Phys. Rev. D 71 (2005) 115001 arXiv:hep-ph/0502162.

[50] R. Casalbuoni, S. De Curtis, D. Dolce and D. Dominici, Phys. Rev. D 71 (2005) 075015 arXiv:hep-ph/0502209.

[51] R. Sekhar Chivukula, E. H. Simmons, H. J. He, M. Kurachi and M. Tanabashi, Phys. Rev. D 72 (2005) 015008 arXiv:hep-ph/0504114.

[52] R. Barbieri, A. Pomarol, R. Rattazzi and A. Strumia, Nucl. Phys. B 703 (2004) 127 arXiv:hep-ph/0405040.

[53] R. S. Chivukula, E. H. Simmons, H. J. He, M. Kurachi and M. Tanabashi, Phys. Lett. B 603 (2004) 210 arXiv:hep-ph/0408262.

[54] R. S. Chivukula, E. H. Simmons, H. J. He, M. Kurachi and M. Tanabashi, Phys. Rev. D 72 (2005) 075012 arXiv:hep-ph/0508147.

[55] R. Sekhar Chivukula, E. H. Simmons, H. J. He, M. Kurachi and M. Tanabashi, Phys. Rev. D 72 (2005) 095013 arXiv:hep-ph/0509110.

[56] R. Sekhar Chivukula, B. Coleppa, S. Di Chiara, E. H. Simmons, H. J. He, M. Kurachi and M. Tanabashi,
Phys. Rev. D 74 (2006) 075011 arXiv:hep-ph/0607124.

[57] B. Coleppa, S. Di Chiara and R. Foadi, JHEP 0705 (2007) 015 arXiv:hep-ph/0612213.

[58] T. Appelquist and C. W. Bernard, Phys. Rev. D 23 (1981) 425.

[59] T. Appelquist and C. W. Bernard, Phys. Rev. D 22 (1980) 200.

[60] A. C. Longhitano, Phys. Rev. D 22 (1980) 1166.

[61] A. C. Longhitano, Nucl. Phys. B 188 (1981) 118.

[62] T. Appelquist and G. H. Wu, Phys. Rev. D 48 (1993) 3235 arXiv:hep-ph/9304240.

[63] M. J. Herrero and E. Ruiz Morales, Nucl. Phys. B 418 (1994) 431 arXiv:hep-ph/9308276.

[64] J. Gasser and H. Leutwyler, Annals Phys. 158 (1984) 142.

[65] J. Gasser and H. Leutwyler, Nucl. Phys. B 250 (1985) 465.

[66] S. Matsuzaki, R. S. Chivukula, E. H. Simmons and M. Tanabashi, Phys. Rev. D 75 (2007) 073002 arXiv:hep-ph/0607191.

[67] R. S. Chivukula, E. H. Simmons, S. Matsuzaki and M. Tanabashi, Phys. Rev. D 75 (2007) 075012 arXiv:hep-ph/0702218.

[68] S. Dawson and C. B. Jackson, Phys. Rev. D 76 (2007) 015014 arXiv:hep-ph/0703299.

[69] R. Barbieri, G. Isidori, V. S. Rychkov and E. Trincherini, arXiv:0806.1624 [hep-ph].

[70] R. Sekhar Chivukula, E. H. Simmons, H. J. He, M. Kurachi and M. Tanabashi, Phys. Rev. D 75 (2007) 035005 arXiv:hep-ph/0612070.

[71] L. Anichini, R. Casalbuoni and S. De Curtis, Phys. Lett. B 348 (1995) 521 arXiv:hep-ph/9410377.

[72] A. Manohar and H. Georgi, Nucl. Phys. B 234 (1984) 189.

[73] A. Birkedal, K. Matchev and M. Perelstein, Phys. Rev. Lett. 94 (2005) 191803 arXiv:hep-ph/0412278.

[74] H. J. He et al., arXiv:0708.2588 [hep-ph].

[75] W. M. Yao et al. [Particle Data Group], J. Phys. G 33 (2006) 1.

[76] E. Accomando, S. De Curtis, D. Dominici and L. Fedeli, arXiv:0807.2951 [hep-ph].

[77] E. Accomando, S. De Curtis, D. Dominici and L. Fedeli, arXiv:0807.5051 [hep-ph].

[78] R. S. Chivukula and E. H. Simmons, arXiv:0808.2071 [hep-ph].

[79] A. Abulencia et al. [CDF Collaboration], Phys. Rev. D 75 (2007) 091101 arXiv:hep-ex/0611022. 